\title{
Crystalline Structure Analysis of All-cellulose Nanocomposite Films Based on Corn and Wheat Straws
}

\author{
Hongxia Bian, Yanyan Yang, and Peng Tu*
}

Cellulose solution and nanocellulose were prepared from corn straw and wheat straw and then used to fabricate an all-cellulose nanocomposites film (ANF). The crystal structure (CS) of ANFs was analyzed by X-ray diffraction (XRD) and Fourier transform infrared spectrometry (FTIR). Cellulose-I and cellulose-II were found to coexist within regenerated cellulose films (RCF) and ANFs. With the change of nanocellulose content, the proportions of cellulose-I and cellulose-II changed. Cellulose transformation was found to depend on the raw material and the preparation method. When cellulose solution was prepared from corn straw that had been extracted, the cellulose type tended to be transformed from cellulose-I to cellulose-II; the proportion of cellulose-I showed a tendency to increase when nanocellulose content exceeded $1.5 \%$. When the dissolved cellulose had been treated by an acidalkali method, the results did not follow a clear pattern. However, when cellulose solution was prepared from wheat straw, under extraction method, the cellulose type tended to transform from cellulose-I to cellulose-II; under acid-alkali method, cellulose-I did not follow a clear pattern with nanocellulose content. Though the small amount of nanocellulose can't dominate the content of cellulose-I, it could cause an increase in disorder of the cellulose matrix.

Keywords: All-cellulose nanocomposite film (ANF); Crystalline index (CI); XRD; FTIR analysis

Contact information: College of Science, Gansu Agricultural University, Lanzhou PR China;

* Corresponding author: tupeng815@163.com

\section{INTRODUCTION}

There is a growing demand for environmentally friendly materials including cellulose, a biodegradable and renewable natural material (Hai et al. 2017). Due to the huge reserve of cellulose, researchers can easily obtain, process, and modify it to produce a variety of cellulosic materials. One such application is films to replace non-degradable packaging materials. Further research on cellulose films will accelerate environmental protection in industrial development and technological advancement (Escursell et al. 2021).

The crystallinity of cellulose is one of its most important attributes, as it reflects many properties of cellulose products, especially mechanical strength ( $\mathrm{Li}$ et al. 2016). Cellulose takes the form of five crystal structures: cellulose-I, II, III, IV, and V (Dong and Gray 1997). Cellulose-I is the main crystal structure in native cellulose, and it has two polymorphs, triclinic structure $\left(\mathrm{I}_{\alpha}\right)$ and a monoclinic structure $\left(\mathrm{I}_{\beta}\right)$. These two polymorphs coexist in various proportions depending on the cellulose source. In certain conditions, cellulose I can become transformed into cellulose II, and mercerization and regeneration are generally two such processes. For example, films made of Valonia ventricose, ramie, and bacterial cellulose and prepared in different ways contain cellulose I (Liang et al. 1959a). The crystal structure (CS) of cellulose powder is transformed from cellulose-I to 
cellulose-II after treating with 5 to $10 \mathrm{wt} \% \mathrm{NaOH}$ (Oh et al. 2005).

Cellulose-I and cellulose-II appear commonly in cellulosic films. Due to the intermolecular and intramolecular hydrogen-bonding of cellulose macro-molecules, the CS of all cellulose materials is stable and rigid. Moreover, the primary hydrogen bonds are $\mathrm{O}_{2}-$ $\mathrm{H}-\mathrm{O}_{6}$ and $\mathrm{O}_{3}-\mathrm{H} \ldots \ldots \mathrm{O}_{5}$ in cellulose- $\mathrm{I}$, and $\mathrm{O}_{2}-\mathrm{H}-\mathrm{O}_{6}, \mathrm{O}_{2}-\mathrm{H}-\mathrm{O}_{2}, \mathrm{O}_{6}-\mathrm{H}-\mathrm{O}_{6}$ in cellulose-II. Two cellulose macro-molecules chains parallel to each other are connected by hydrogen bonds to form a three-dimensional network structure (Yue 2011). The CI of cellulosic materials and cellulose derivatives is higher than most other straight-chain polymers. Therefore, studies on cellulose CI and CS in cellulose films will improve their mechanical properties.

X-ray diffraction (XRD) and Fourier transform infrared spectrometry (FTIR) have been used to analyze all-cellulose nanocomposite films (ANFs) and regenerated cellulose films (RCFs) (Angelin et al. 2017). Due to the advantage of rapid detection, simple operation, and convenient data processing, XRD has a wide range of applications. The XRD spectrum information can identify the crystal structure of the material and determine the orientation of crystal. With the advantages of fast analysis and simple operation, FTIR can simultaneously determine a variety of functional groups; with the advantages of good selectivity, high sensitivity, and less sample damage, it can determine the crystal type of cellulose film by analysis of the shifting of characteristic peaks position in the spectrum.

There are several ways used to calculate the CI of cellulose, usually involving XRD and FTIR spectra. The methods to obtain CI based on X-ray diffraction (CI(XD)) include the empirical method of Segal et al. (1959) and Jayme and Knolle (1964), the area ratio between crystalline and amorphous phase in XRD curve (Zavadskii 2004), and the splitting diffraction peak by Lorentzian function (Wang et al. 2010). The CI obtained by FTIR (CI(IR)) is obtained by absorbance ratios, such as $A_{1431,1419} / A_{897,} 894$ and $A_{1263} / A_{1202,1200}(\mathrm{Oh}$ et al. 2005). The empirical method using the ratio of intensity of diffractogram peak at the position of the crystal plane $\left(\begin{array}{lll}0 & 0 & 2\end{array}\right)\left(2 \theta=22.6^{\circ}\right.$ for cellulose-I and $2 \theta=21.7^{\circ}$ for celluloseII) and amorphous background ( $2 \theta=19.0^{\circ}$ for cellulose-I, $2 \theta=16.0^{\circ}$ for cellulose-II) can be used to calculate the CI as follows (Nelson et al. 1964b),

$$
C I \%=\frac{I_{002}-I_{a m}}{I_{002}} \times 100
$$

where $I_{002}$ is the diffraction intensity of $\left(\begin{array}{lll}0 & 0 & 2\end{array}\right)$ crystal plane, and $I_{\mathrm{am}}$ is the scattering intensity of amorphous region.

The $\mathrm{CI}(\mathrm{XD})$ also can be calculated from the ratio of the diffraction peak area of cellulose-I (or cellulose-II) and total peak area. Using the pure sample method for calculating CI, a diffraction peak intensity of $100 \%$ crystalline sample $\left(I_{\mathrm{c} 100}\right)$ of the substance (or $100 \%$ amorphous sample, $I_{\mathrm{a}} 100$ ) and diffraction peak intensity of the sample can be measured, and the values of $I_{\mathrm{c} 100}\left(I_{\mathrm{a} 100}\right)$ and $I_{\mathrm{c}}\left(I_{\mathrm{a}}\right)$ can be characterized, respectively. The relationship is shown by the following equation (Huang and Li 2012),

$$
C I \%=\frac{I_{C}}{I_{c 100}} \times 100
$$

where $I_{\mathrm{c} 100}$ is the diffraction intensity of $100 \%$ crystalline sample, $I_{\mathrm{c}}$ is the diffraction intensity of measured sample, $I_{\mathrm{a} 100}$ is the scattering intensity of $100 \%$ amorphous sample, and $I_{\mathrm{a}}$ is the scattering intensity of amorphous region at measured sample.

Nanocellulose typically retains the crystal structure of cellulose I and has unique properties related to its size. In all-cellulose composite, it is proposed that the addition of nanocellulose at a sufficiently high level might allow it to remain in cellulose-I form or alternatively, it may be partly or fully converted to cellulose-II as a consequence of partial 
dissolution. In the study of Zhao (2014), the concentration of cellulose I increased when the proportion of nanocellulose was increased from $5 \%$ to $20 \%$. On the other hand, Gindl and Keckes (2005) showed that cellulose I could be dissolved and transformed into cellulose II in LiCl/DMAc solution.

In this study, cellulose and nanocellulose derived from wheat straw and corn straw agricultural residues were used to prepared ANFs and RCFs. The cellulose, nanocellulose, ANFs, and RCFs were prepared as previously described (Bian et al. 2020). Based on the different materials and preparation methods, the crystallinities of ANFs and RCFs were compared. Because the CS and CI of a material is closely related to its mechanical properties, research on the CS and CI of cellulose products is important for expanding the use of cellulose-based materials.

\section{EXPERIMENTAL}

\section{Materials}

Cellulose from corn and wheat straw agricultural residues was prepared using an acid-alkali method and an extraction treatment, respectively. Nanocellulose, which also came from corn and wheat straws, was prepared by the TEMPO oxidation method. The DMAc (99.8\%) and $\mathrm{LiCl}$ (Shanghai Zhanyun Chemical Industry Co. Ltd, Shanghai) were used to dissolve cellulose.

\section{Methods}

Cellulose, nanocellulose, and all-cellulose nanocomposite films were prepared as described by Bian et al. (2020). The brief preparation methods are as follows. The cellulose was prepared by acid-alkali and extracting methods, respectively. The acid-alkali method uses acid and alkali solutions to obtain cellulose, with ultrasonication (500 W, $30 \mathrm{~min}$ ) as an auxiliary. The extracting method requires a Soxhlet extractor to remove any fat, wax, etc. The straw was extracted for $6 \mathrm{~h}$ in the mixed solution of toluene and alcohol with volume ratio of $2: 1$ at $90{ }^{\circ} \mathrm{C}$. Nanocellulose then was prepared by TEMPO oxidation method, which introduces a lot of carboxyl groups. The diameters of particles of two nanocelluloses were both in the range 20 to $50 \mathrm{~nm}$. The lengths were $50 \mathrm{~nm}$ to $2 \mu \mathrm{m}$ for wheat stalk and $100 \mathrm{~nm}$ to $10 \mu \mathrm{m}$ for corn stalk, as determined from atom force microscope (AFM) images (Bian et al. 2020). ANFs were prepared by regenerating the cellulose fibers, which were dissolved using the $\mathrm{DMAc} / \mathrm{LiCl}$ solution system, with the subsequent addition of nanocellulose at the $0.1 \%, 1.5 \%$, and $3.0 \%$ levels, respectively, for strengthening. In the process of fabricating ANF, cellulose fibers and nanocellulose came from the same straw; if cellulose fibers came from wheat straw, nanocellulose also came from wheat straw.

\section{X-ray Diffraction}

An X-ray diffractometer (Puxitongyun XD-2, Beijing, China) with nickel-filtered $\mathrm{Cu} \mathrm{Ka}$ radiation $(\lambda=1.540 \mathrm{~nm})$ was used. The operating voltage and current were $40 \mathrm{kV}$ and $30 \mathrm{~mA}$, respectively; the scanning step and range were $0.01^{\circ} \mathrm{min}^{-1}$ and 10 to $45^{\circ}$, respectively.

\section{Fourier Transform Infrared Spectrometry}

A Thermo Fisher Nicolet IS 50 (Shanghai, China) was used to collect FTIR spectra. 
The characteristic peak ratios were used to obtain CI values. All spectra were recorded with an accumulation of 64 scans, resolution of $4 \mathrm{~cm}^{-1}$, in the range from 4000 to $400 \mathrm{~cm}^{-1}$. Three different measurements for each sample were evaluated, and the average value was calculated.

\section{RESULTS AND DISCUSSION}

\section{XRD Analysis}

All measurement results of XRD were fitted by the peak separation method (Park et al. 2010). The XRD diffraction patterns of ANFs and RCFs prepared from corn straw via the extracting and acid-alkali methods and adding different concentration of nanocellulose are shown in Fig. 1. The diffraction pattern range of ANFs and RCFs is mainly from $10^{\circ}$ to $30^{\circ}$. Cellulose-I is the main cellulose type in nature plant cellulose

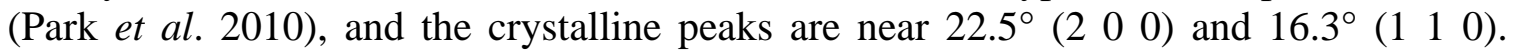
Cellulose-II is also known as man-made fiber, and the crystalline peak is near $20.0^{\circ}(11$ 0 ) and $22.0^{\circ}(200)$ (Kargarzadeh et al. 2017). The diffraction peaks are at $22.3^{\circ}$ and $16.7^{\circ}$ in the corn straw powder, and they are at $22.5^{\circ}$ and $16.3^{\circ}$ in nanocellulose prepared by corn straw (Bian et al. 2020). In Fig. 1a, the crystal peaks appear at $2 \theta=16.1^{\circ}$ to $17.5^{\circ}\left(\begin{array}{lll}1 & 0 & 1\end{array}\right)$ and $2 \theta=20.8^{\circ}$ to $21.9^{\circ}$, indicating the coexistence of cellulose-I and cellulose-II in ANFs and RCFs (Nishiyama et al. 2002; Zhao et al. 2014). Compared with the primary and secondary diffraction peaks at $2 \theta=21.0^{\circ}$ and $16.5^{\circ}$ of RCF, the primary and secondary diffraction peaks of $2 \theta=21.9^{\circ}$ and $17.5^{\circ}$ shifted right when the concentration of nanocellulose was $0.1 \%$ in the ANF. This result indicated that the diffraction lattice planes of cellulose-I were changed, and cellulose-II was increased gradually, based on the observation that the intensity of diffraction peak increased at $21.9^{\circ}$ (Wang et al. 2019). This may be caused by the formation of holes in the sample after ultrasonic treatment during the preparation of cellulose, and the formation of a certain molecular orientation due to a small amount of nanocellulose, which accelerates the conversion of cellulose-I (Bian et al. 2020). However, the intensity of the diffraction peak at $16.1^{\circ}$ and $16.2^{\circ}$ was higher than at $20.8^{\circ}$ and $21.6^{\circ}$ when the concentration of nanocellulose reached $1.5 \%$ and $3.0 \%$. It could be caused by adding nanocellulose.

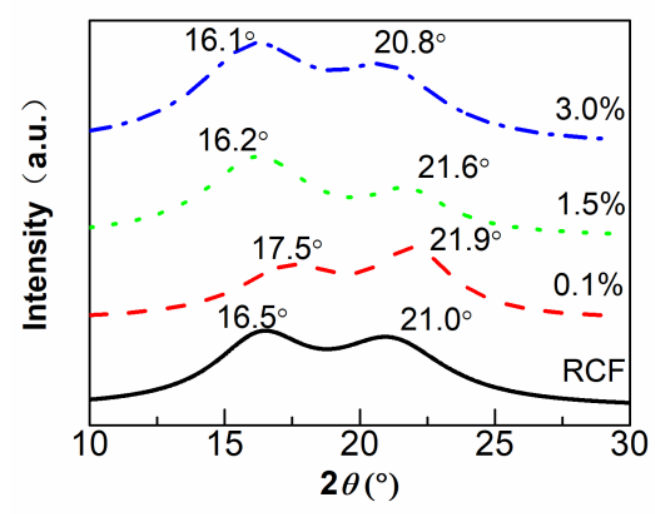

a

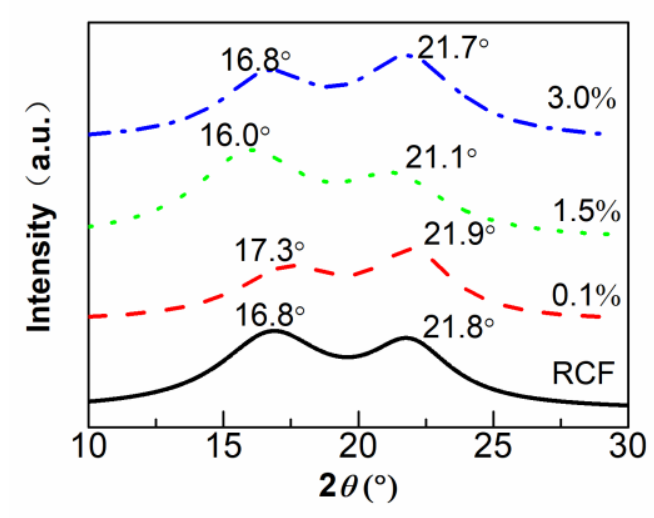

b

Fig. 1. XRD diffraction pattern of ANFs and RCFs which original material came from corn straw and the cellulose were extracted by a) extracting method and b) acid-alkali method 
When the cellulose was prepared by the acid-alkali method, the diffraction pattern of ACFs and RCF was as shown in Fig. 1b. Compared with the diffraction patterns of the ANFs and RCF with cellulose prepared by extracting, the primary and secondary peaks at $2 \theta=16.8^{\circ}, 21.8^{\circ}$ appeared in RCF, and this indicated the coexistence of cellulose-I and cellulose-II. This result was consistent with the crystal type of ANF prepared by extracting. When the concentration of nanocellulose was $0.1 \%$, the peaks at $17.3^{\circ}$ suggested that the diffraction lattice planes of cellulose-I changed, and the higher intensity of diffraction peak at $21.9^{\circ}$ indicated an increased proportion of cellulose-II. The crystal type of ANF did not change and remained in a state of coexistence when the concentration of nanocellulose was $1.5 \%$. The proportion of cellulose-I was found to increase when the nanocellulose concentration was $1.5 \%$; this was evident from an increasing intensity of the diffraction peak at $16.0^{\circ}$. Furthermore, when the concentration of nanocellulose was $3.0 \%$, the intensity of the peak at $2 \theta=21.7^{\circ}$ increased, indicating that the content of cellulose-II was increased. This result could be because that cellulose was soaked by sodium hydroxide $(8 \%)$ during preparation, and alkali treatment converts cellulose-I to cellulose-II. In the process of mercerization, the whole fiber was transformed into the swollen state. The assembly and direction of microfibers were broken, and the parallel chain structure of cellulose-I was transformed into the anti-parallel chain structure of cellulose-II (Manjunath and Peacock 1972). Meanwhile, the results above also indicated that the small amount nanocellulose was not able to increase the cellulose-I in the ANF with the cellulose prepared by the acid-alkali method.

The XRD diffraction patterns of ANFs and RCFs prepared from wheat straw and via extracting and acid-alkali method, respectively, are shown in Fig. 2. In experiment, it was found that the diffraction peaks were at $22.3^{\circ}$ and $16.3^{\circ}$ for the wheat straw powder, and they were at $22.6^{\circ}$ and $17.5^{\circ}$ for nanocellulose prepared by wheat straw. The diffraction pattern of the ANFs ranged from $10^{\circ}$ to $30^{\circ}$. As shown in Fig. 2a, the appearance of the primary peak and the secondary peak at $2 \theta=17.2^{\circ}, 2 \theta=20.6^{\circ}$, respectively, indicated the coexistence of cellulose-I and cellulose-II. When the concentration of nanocellulose was $0.1 \%$, the crystalline type and the intensity of diffraction peak didn't obviously change. Though when the concentration of nanocellulose increased, the crystal type of ANFs also didn't change. The intensity of primary peaks at $2 \theta=21.2^{\circ}$ to $21.3^{\circ}$ was slightly higher than the secondary peaks at $2 \theta=16.6^{\circ}$ to $16.8^{\circ}$. This result suggests that the structure of cellulose-I of ANFs was destroyed by alkalization with ultrasound, and this increased the transformation in which the part of the crystalline region was rearranged into cellulose-II.

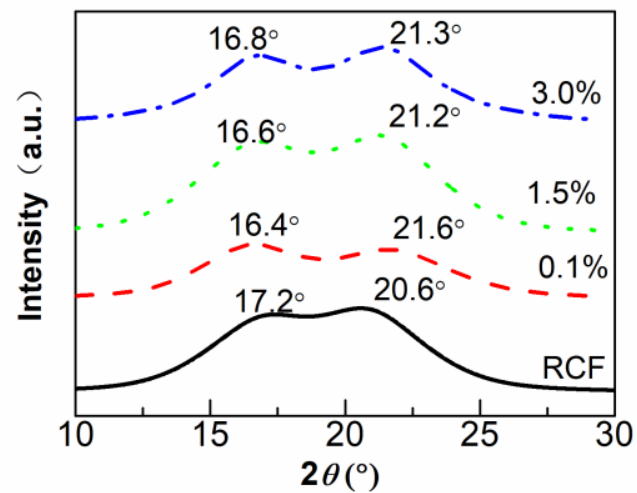

a

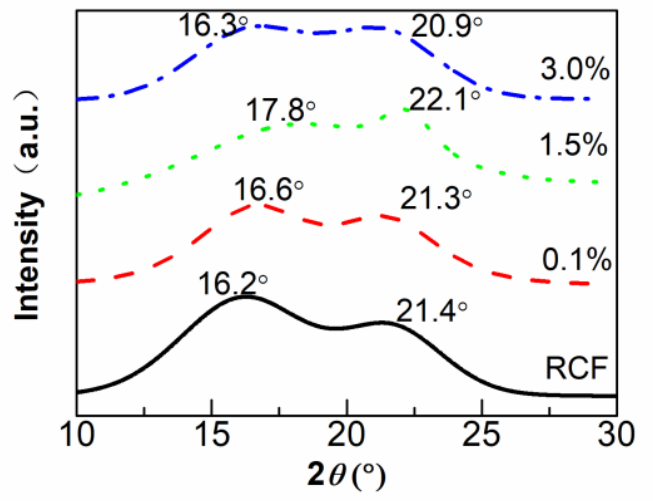

b

Fig. 2. XRD diffraction pattern of ANFs and RCFs which original material came from wheat straw and the cellulose were extracted by a) extracting method and b) acid-alkali method 
As shown in Fig. 2b, compared with the diffraction patterns of ANFs and RCFs from corn straw, the diffraction pattern in ANFs of wheat straw was different. The primary and secondary peaks at $2 \theta=16.2^{\circ}, 21.4^{\circ}$ confirmed that cellulose-I and cellulose-II were both present in RCF. When the concentration of nanocellulose was $0.1 \%$, the crystal type of ANF was consistent with RCF. When the concentration of nanocellulose reached $0.1 \%$, the primary peak at $2 \theta=22.1^{\circ}$ appeared, and the intensity of diffraction peak was higher than the peak at $17.8^{\circ}$. When the concentration of nanocellulose reached $1.5 \%$, the primary peak at $2 \theta=22.1^{\circ}$ appeared and the intensity of diffraction peak was higher the peak at $17.8^{\circ}$. This result demonstrated a tendency for the proportion of cellulose-II to be increased and for the cellulose-I to shift its lattice plane in ANF due to the added nanocellulose. However, when the concentration of nanocellulose reached $3.0 \%$, the primary and secondary peaks at $2 \theta=20.9^{\circ}, 16.3^{\circ}$ appeared and had almost the same intensity. Though the increase of nanocellulose concentration didn't raise the content of cellulose-I at lower concentration, it could cause an increase in cellulose matrix disorder. Nanoparticle can cause the changes of crystallization behavior and crystal structure in the polymer, and the changes are affected by the dispersion (Wang et al. 2006).

\section{FTIR Analysis}

The FTIR spectra of RCFs and ANFs with different concentrations of nanocellulose and cellulose extracted from corn straw are presented Fig. 3. As shown in Fig. 3a, the bands at $3347 \mathrm{~cm}^{-1}$ assigned as $-\mathrm{OH}$ stretching vibration is shifted to lower wavenumbers 3338 $\mathrm{cm}^{-1}$. The XRD patterns of the RCF of corn straw prepared by extracting show that cellulose-I and cellulose-II are present. There is a wide absorptive peak at $3338 \mathrm{~cm}^{-1}$, which also demonstrates that cellulose-I and cellulose-II were coexisting in the RCF. The band at $2891 \mathrm{~cm}^{-1}$ is assigned as -CH stretching in cellulose-II (Higgins et al. 1961). The absorption peak gradually changed from 2901 to $2891 \mathrm{~cm}^{-1}$ and even $2890 \mathrm{~cm}^{-1}$ with the increasing of nanocellulose concentration. The band at $2901 \mathrm{~cm}^{-1}$ is assigned as $-\mathrm{CH}$ stretching in cellulose-I (Oh et al. 2005). This phenomenon indicates a gradual transition from celluloseI to cellulose-II. Otherwise, the absorption band was lacking in the range from 1600 to $1750 \mathrm{~cm}^{-1}$. This is why that region is not listed in the subsequent figures.

As shown in Fig. 3b, the absorbance intensity at $1419 \mathrm{~cm}^{-1}$ assigned as symmetric $-\mathrm{CH}_{2}$ bending, was shifted to higher wavenumbers $\left(1421 \mathrm{~cm}^{-1}\right)$ when the concentration of nanocellulose was $1.5 \%$, indicating the tendency for cellulose-I to increase (Liang et al. $1959 \mathrm{~b})$. With increasing nanocellulose concentration, the intensity of the absorption peak at $1367 \mathrm{~cm}^{-1}$ increased, and the width of the absorption peak gradually narrowed. The effect may be caused by the content of cellulose-I in the CS of ANFs increase due to the addition of nanocellulose. The band at $1262 \mathrm{~cm}^{-1}$ is assigned as $-\mathrm{COH}$ in the plane at C-2 and C-3 in cellulose-I and cellulose-II, and it indicates the coexistence of cellulose-I and cellulose-II in ANFs and RCFs. The bands at $1157 \mathrm{~cm}^{-1}$ and $1015 \mathrm{~cm}^{-1}$ are assigned as C-O-C stretching in cellulose-II and C-O stretching, respectively. Similarly, with the increase of nanocellulose content, these peaks changed from flat to sharp; the region of cellulose-II in the CS of ANFs gradually concentrates in a certain part. The band at $896 \mathrm{~cm}^{-1}$ is assigned as $\beta$-glycosidic linage for cellulose-I, and it was shifted to $894 \mathrm{~cm}^{-1}$ when the concentration of nanocellulose was $0.1 \%$. This result indicates that the crystalline type of ANFs changed from cellulose-I to cellulose-II. The band at $992 \mathrm{~cm}^{-1}$ assigned C-O stretching at C-6 was shifted to the lower wavenumber at $990 \mathrm{~cm}^{-1}$, and the absorption band moved from 894 to $895 \mathrm{~cm}^{-1}$ when the concentration of nanocellulose was $3.0 \%$. There is the tendency that cellulose-I increase and the fluctuations at bands 992 and $894 \mathrm{~cm}^{-1}$ could be the competition 
between the cellulose-I in nanocellulose and the disorder while adding nanocellulose.

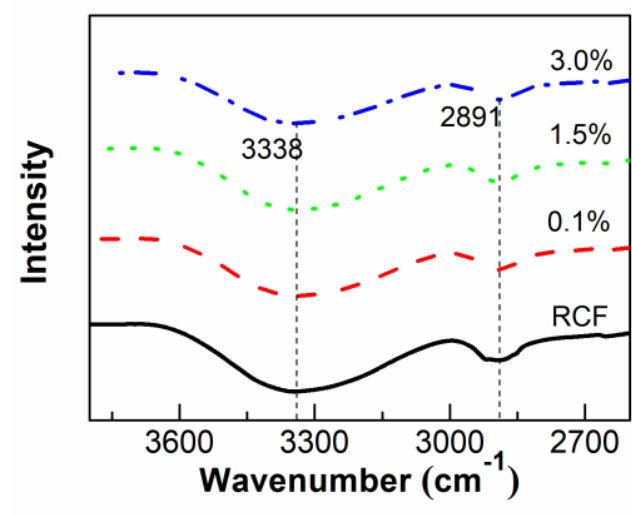

a

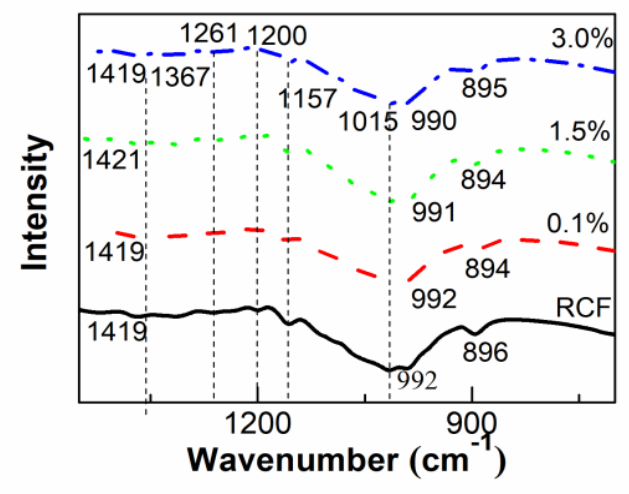

b

Fig. 3. FTIR spectra of ANFs and RCFs which original material came from corn straw and the cellulose were prepared by extracting method a) 3800 to $2600 \mathrm{~cm}^{-1}$, b) 700 to $1450 \mathrm{~cm}^{-1}$

The FTIR spectra of RCFs and ANFs with different nanocellulose concentration and the cellulose solution was prepared with the acid-alkali method from corn straw are shown in Fig. 4. In Fig. 4a, there is a large absorption peak between 3643 and $3013 \mathrm{~cm}^{-1}$. The absorption intensity of peak reached the maximum when the position was $3322 \mathrm{~cm}^{-1}$. There is also a broadening absorption peak between 2977 and $2784 \mathrm{~cm}^{-1}$. The absorption intensity of peak reached the maximum when the position was $2894 \mathrm{~cm}^{-1}$. The bands at 3322 and $2894 \mathrm{~cm}^{-1}$ are assigned as $-\mathrm{OH}$ stretching vibration and $-\mathrm{CH}$ stretching in cellulose-II, respectively (Yukako et al. 2017).

As shown in Fig. 4b, the absorbance intensity at $1419 \mathrm{~cm}^{-1}$ is assigned as symmetric $-\mathrm{CH}_{2}$ bending of cellulose-II. The peak at $1422 \mathrm{~cm}^{-1}$ was shifted to $1420 \mathrm{~cm}^{-1}$ when the concentration of nanocellulose was $0.1 \%$, which indicated that the crystalline type of RCF gradually was transformed to cellulose-II. The band at $1317 \mathrm{~cm}^{-1}$ was assigned as $-\mathrm{CH}_{2}$ wagging at C-6. The position of the peaks at $1315 \mathrm{~cm}^{-1}$ appeared as a weak absorption peak in RCF, which suggests that cellulose-I and cellulose-II both were present in RCF. When the concentration of nanocellulose was $0.1 \%$ and $1.5 \%$, the peak at $1315 \mathrm{~cm}^{-1}$ was shifted to $1312 \mathrm{~cm}^{-1}$, which might be due to the use of ultrasonic treatment. The CS was destroyed partially and reconstituted into cellulose-II. The band at $993 \mathrm{~cm}^{-1}$ shifted to $990 \mathrm{~cm}^{-1}$ when the concentration of nanocellulose was $0.1 \%$. The absorbance intensity at $895 \mathrm{~cm}^{-1}$ was assigned as antisymmetrical out of phase stretching of cellulose-II, and the peak located at $895 \mathrm{~cm}^{-1}$ shifted to $897 \mathrm{~cm}^{-1}$ when the concentration of nanocellulose was $1.5 \%$. These indicate the tendency for the proportion of cellulose-I to increase. The effect could be caused by the competition between cellulose-I in nanocellulose and disorder in cellulose matrix after adding nanocellulose. Similarly, when the concentration of nanocellulose was $3.0 \%$, the absorbance peaks at $1420,1312,990$, and $897 \mathrm{~cm}^{-1}$ were shifted to higher wavenumbers at $1422,1315,991,895 \mathrm{~cm}^{-1}$. This provides evidence that cellulose-I became transformed to cellulose-II in ANFs. Referring to XRD diffraction results, the cellulose-I in lower concentration of nanocelluloses can't dominate the CS of ANFs. 


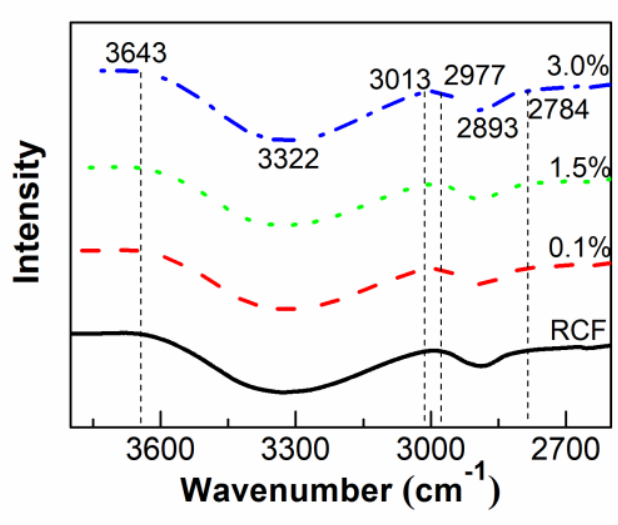

a

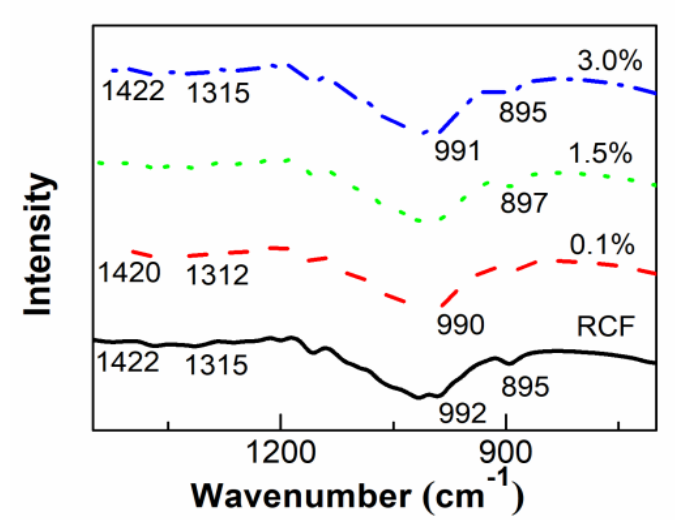

b

Fig. 4. FTIR spectra of ANFs and RCFs which original material came from corn straw and the cellulose were prepared by acid-alkali method a) 3800 to $2600 \mathrm{~cm}^{-1}$, b) 700 to $1450 \mathrm{~cm}^{-1}$

The FTIR spectra of RCFs and ANFs with different concentration nanocellulose and cellulose treated by extracting method from wheat straw are shown in Fig. 5. From Fig. 5a, the bands at $3346 \mathrm{~cm}^{-1}$ show -OH stretching of intramolecular hydrogen bonds, indicating cellulose-I in ANFs obtained from wheat straw. The bands at $2891 \mathrm{~cm}^{-1}$ represent $-\mathrm{CH}$ stretching of cellulose-II. The peak at $2916 \mathrm{~cm}^{-1}$ is assigned as $-\mathrm{CH}$ stretching (Liang et al. 1959a).

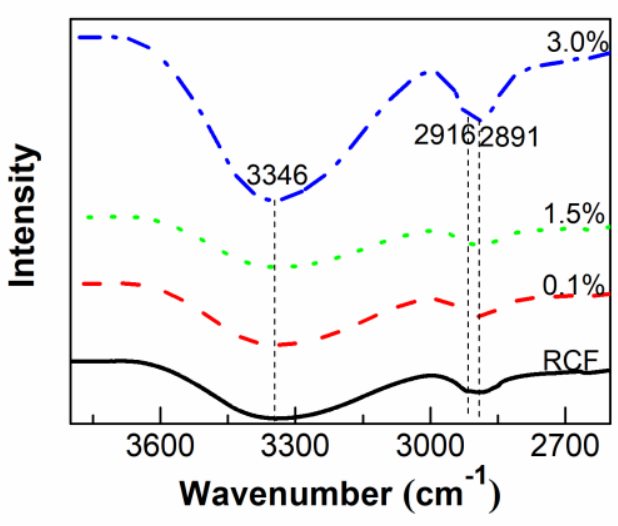

a

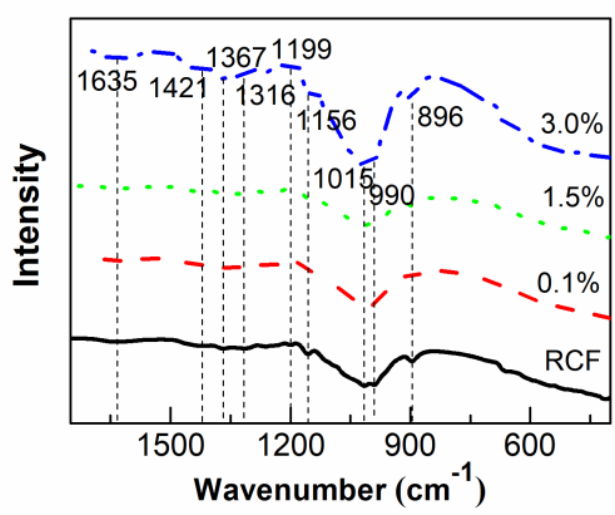

b

Fig. 5. FTIR spectra of ANFs and RCFs with different concentration nanocellulose and cellulose treated by extracting method from wheat straw a) $3800-2600 \mathrm{~cm}^{-1}$, b) $400-1750 \mathrm{~cm}^{-1}$

As shown in Fig. 5b, the band at $1635 \mathrm{~cm}^{-1}$ is assigned as $-\mathrm{OH}$ bending in water. The bands at 1421, 1367, and $1316 \mathrm{~cm}^{-1}$ are assigned as $-\mathrm{CH}_{2}$ bending, $-\mathrm{CH}$ bending, and $-\mathrm{CH}_{2}$ wagging at $\mathrm{C}-6$; these findings indicate that cellulose-I and cellulose-II coexist in ANF and RCF (Nelson et al. 1964a). The absorption peaks located at $1199 \mathrm{~cm}^{-1}$ are assigned as $-\mathrm{COH}$ bending at $\mathrm{C}-6$ in cellulose-II. The absorbance intensity at $1156 \mathrm{~cm}^{-1}$ is assigned as $\mathrm{C}-\mathrm{O}-\mathrm{C}$ stretching vibration in cellulose-II. The absorption peaks at 1015 and $990 \mathrm{~cm}^{-1}$ are assigned as C-O stretching at C-6 in cellulose-II. The bands at $896 \mathrm{~cm}^{-1}$ are assigned as -COC at $\beta$-glycosidic linkage in cellulose-I. When the concentration of nanocellulose was $3.0 \%$, it is worth noting that the intensity of absorption peaks all increased. 
The FTIR spectra of RCFs and ANFs with different concentration nanocellulose and cellulose treated by acid-alkali method from wheat straw are shown in Fig. 6. From Fig. 6a, the bands at $3338 \mathrm{~cm}^{-1}$ shows $-\mathrm{OH}$ stretching of intramolecular hydrogen bonds. This data indicates that cellulose-I was present in ANFs, which were obtained from wheat straw. The bands at $2891 \mathrm{~cm}^{-1}$ manifests $-\mathrm{CH}$ stretching of cellulose-II. The position of peak at $2916 \mathrm{~cm}^{-1}$ disappeared when the concentration of nanocellulose was $1.5 \%$ and $3.0 \%$.

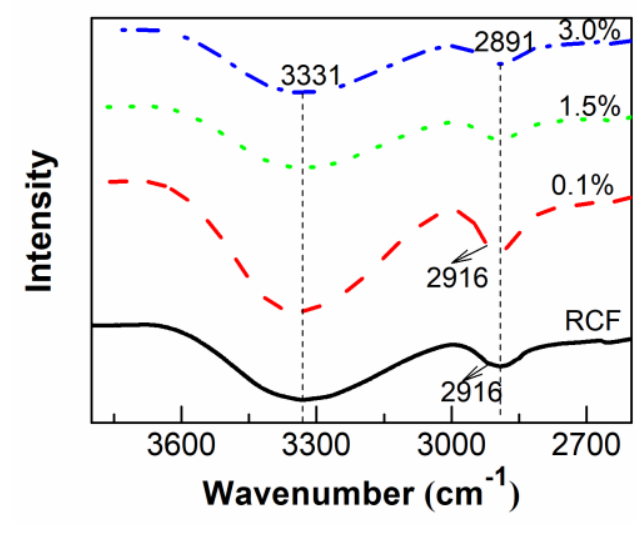

a

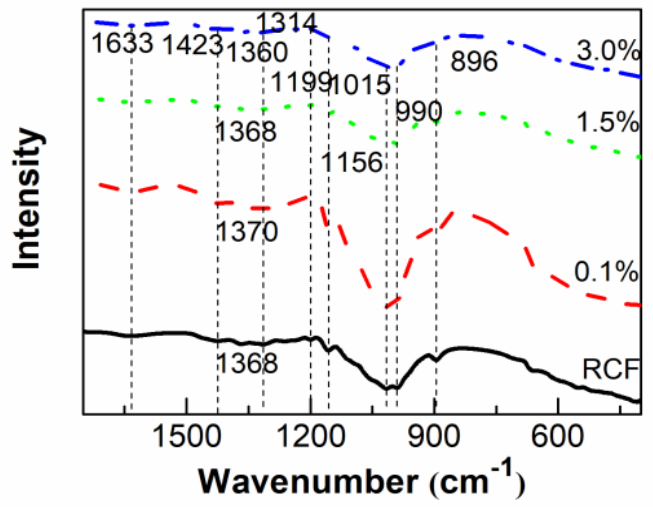

b

Fig. 6. FTIR spectra of ANFs and RCFs with different concentration nanocellulose and cellulose treated by acid-alkali method from wheat straw a) 3800 to $2600 \mathrm{~cm}^{-1}$, b) 400 to $1750 \mathrm{~cm}^{-1}$

As shown in Fig. 6b, the band at $1633 \mathrm{~cm}^{-1}$ is assigned as $-\mathrm{OH}$ bending in water. The bands at $1423 \mathrm{~cm}^{-1}$ are assigned as $-\mathrm{CH}_{2}$ bending and indicate that cellulose-I was present in ANFs and CRF. The peak at $1368 \mathrm{~cm}^{-1}$ in CRF was shifted to $1370 \mathrm{~cm}^{-1}$ when the concentration of nanocellulose was $0.1 \%$. This phenomenon suggests that crystalline type was transformed from cellulose-I to cellulose-II. When the concentration of nanocellulose was increased from $0.1 \%$ to $1.5 \%$ and then to $3.0 \%$, the absorption peak also was shifted from 1370 to $1368 \mathrm{~cm}^{-1}$ and then to $1360 \mathrm{~cm}^{-1}$. This indicates that the nanocellulose content resulted in an increase in the $-\mathrm{CH}$ bending absorbance, which is associated with cellulose-I. The bands at $1314 \mathrm{~cm}^{-1}$ are assigned as $-\mathrm{CH}_{2}$ wagging at C-6, and they indicate that cellulose-II was present in ANF and CRF. The absorption peaks at $1199 \mathrm{~cm}^{-1}$ are assigned as $-\mathrm{COH}$ bending at C-6 in cellulose-II. The absorbance intensity at $1156 \mathrm{~cm}^{-1}$ is assigned as $\mathrm{C}-\mathrm{O}-\mathrm{C}$ stretching vibration in cellulose-II. The absorption peaks at $1015 \mathrm{~cm}^{-1}$ and $990 \mathrm{~cm}^{-1}$ are assigned as C-O stretching at C-6 in cellulose-II. The bands at $896 \mathrm{~cm}^{-1}$ are assigned as $-\mathrm{COC}$ at $\beta$-glycosidic linkage in cellulose-I. When the concentration of nanocellulose was $0.1 \%$, the intensity of all absorption peaks increased.

\section{Crystallinity Index (Cl) Analysis}

In this paper, the calculation equation of CI on X-ray diffraction was as follows,

$$
C I \%=\frac{A}{B} \times 100
$$

where $A$ is the area of crystalline peaks, and $B$ is the area of all peaks (crystalline and amorphous).

The CI values of RCFs and ANFs are shown in Table 1. The CI of RCF where cellulose was prepared by acid-alkali method from corn straw was $63.6 \%$, which was 
higher than the RCF where cellulose was prepared by extracting from corn straw (Bian et al. 2020). The CI of RCF obtained from wheat straw was the same. In the ANFs which cellulose prepared by acid-alkali method from corn straw, the CI increased with increasing nanocellulose concentration. When the nanocellulose concentration was $3.0 \%$, the CI of ANF was $67.9 \%$. In the ANFs which cellulose prepared by extracting method from corn straw, when the ANFs with concentrations of $0.1 \%$ and $1.5 \%$ nanocellulose, the CI increased to $71.9 \%$. However, when the concentration of nanocellulose was $3.0 \%$, the CI of ANF decreased to $58.6 \%$.

In the ANFs in which cellulose had been prepared by the acid-alkali method from wheat straw, the opposite phenomenon occurred. The CI decreased to $52.1 \%$, when the nanocellulose content was $3.0 \%$. This may be because the ANFs absorbed $\mathrm{H}_{2} \mathrm{O}$ molecules in the storage process, and $\mathrm{H}_{2} \mathrm{O}$ molecules gradually entered the crystalline region from the amorphous region, destroying the part of crystalline structure and reducing the CI of ANFs (Agarwal et al. 2017). When the concentration of nanocellulose was 3.0\%, the CI of ANFs with cellulose prepared by extractimg from wheat straw increased to $63.9 \%$.

The CI of nanocellulose from corn and wheat stalk that were prepared in the authors' lab were $76.1 \%$ and $57.5 \%$, respectively. The CI of nanocellulose from corn stalk was $72.6 \%$ (Mtibe et al. 2015), and the CI of nanocellulose from wheat stalk was a range from $50.0 \%$ to $77.8 \%$ (Alemdar and Sain 2008), and the difference was usually caused by method of preparation. Though nanocellulose showed a high CI, the CI of ANFs didn't show regular increase with nanocellulose, and even the CI of ANFs was lower than what might be theoretically established in the study. These results could be caused by the small content and small size of nanocellulose.

Table 1. The $\mathrm{Cl}$ of All Kinds of ANFs

\begin{tabular}{|c|c|c|c|c|c|}
\hline \multirow{2}{*}{ Material } & Method of Preparing & & \multicolumn{3}{|c|}{ Nanocellulose Content } \\
\cline { 3 - 6 } & Cellulose & RCF & $0.1 \%$ & $1.5 \%$ & $3 \%$ \\
\hline \multirow{2}{*}{ Corn straw } & Acid-alkali method & 63.6 & 59.6 & 64.7 & 67.9 \\
\cline { 2 - 6 } & Extracting method & 58.5 & 68.0 & 71.9 & 58.6 \\
\hline \multirow{2}{*}{ Wheat straw } & Acid-alkali method & 72.7 & 60.9 & 58.5 & 52.1 \\
\cline { 2 - 6 } & Extracting method & 56.9 & 66.9 & 58.1 & 63.9 \\
\hline
\end{tabular}

\section{CONCLUSIONS}

1. The crystal structure (CS) and crystallinity index (CI) were determined for all-cellulose nanocomposite films (ANFs), which were fabricated using the cellulose and nanocellulose extracted from wheat and corn straw. It was found that the preparation method of cellulose solutions and addition of nanocellulose were the key influencing factors affecting the CS and CI in ANFs.

2. In different ANFs, the transformations of crystal structure exhibited differences with increasing of nanocellulose. The CS of all ANFs for which cellulose was obtained from corn straw exhibited transformation from cellulose-I to cellulose-II when the concentration of nanocellulose was $0.1 \%$, but when the concentration increased to $3.0 \%$, the cellulose-I present in the resulting film increased. In the ANFs for which cellulose was obtained by an extraction method from wheat straw, the CS was only transformed from cellulose-I to cellulose-II when the concentration of nanocellulose was $0.1 \%$, but in the ANFs for which cellulose was obtained by acid-alkali method 
from wheat straw, the proportion of cellulose-I in the resulting ANFs increased when the concentration of nanocellulose was $1.5 \%$. It is worth noting that in the FTIR spectra, the bands at $3347,1419,992,895 \mathrm{~cm}^{-1}$ were shifted to $3338,1421,990,894 \mathrm{~cm}^{-1}$, respectively; this indicated that cellulose-I was increased in ANFs for which cellulose was obtained by the extraction method from corn straw. The absorption peaks at 1420 , 1312,990 , and $897 \mathrm{~cm}^{-1}$ were shifted to higher wave numbers at 1422, 1315, 991, and $895 \mathrm{~cm}^{-1}$, respectively, and this suggested that cellulose-I was transformed to celluloseII in ANFs for which cellulose was obtained by acid-alkali method from corn straw. The appearance of absorption peaks at 3346, 2891, 2916, 1421, 1367, 1316, $896 \mathrm{~cm}^{-1}$ indicated the coexistence of cellulose-I and cellulose-II in wheat straw films. This could be caused by adding nanocellulose (such that cellulose-I would be expected to increase), but the above results also shown that the lower concentration of nanocelluloses can't dominate the CS of ANFs.

3. Calculating the CI of ANFs, the CI of RCF obtained from corn straw prepared by the acid-alkali method was $63.6 \%$, which was higher than that the cellulose prepared by extracting of corn straw. The CI of RCF obtained from wheat straw was also the same. In the ANF of corn straw which prepared by acid-alkali method, the CI of ANF increased to $67.9 \%$ when the concentration of nanocellulose increased to $3.0 \%$. The CI of ANFs didn't show regular increase with nanocellulose. This could be due to the small content and small size of nanocellulose.

\section{ACKNOWLEDGEMENTS}

The authors are grateful to Gansu Agricultural University, Lanzhou, China for supporting this research by providing fabrication and measurement facilities. This work was also funded by the Youth Doctoral Fund of Higher Education in Gansu Province (2021QB-032)

\section{REFERENCES CITED}

Agarwal, U. P., Ralph, S. A., Baez, C., Reiner, R. S., and Verrill, S. P. (2017). "Effect of sample moisture content on XRD-estimated cellulose crystallinity index and crystallite size," Cellulose 24(5), 1971-1984. DOI: 10.1007/s10570-017-1259-0

Alemdar, A., and Sain, M. (2008). "Isolation and characterization of nanofibers from agricultural residues - Wheat straw and soy hulls," Bioresource Technology 99, 1664-1671. DOI: 10.1016/j.biortech.2007.04.029

Angelin, V., P., Sangeetha, K. Thandapani, G., Sudha, P. N., Jayachandran, V., and Sukumaran A. (2017). "FTIR, XRD and DSC studies of nanochitosan, cellulose acetate and polyethylene glycol blend ultrafiltration membranes," International Journal of Biological Macromolecules 104(Pt B), 1721-1729. DOI: 10.1016/j.ijbiomac.2017.03.122

Bian, H. X., Tu, P., and Chen, J. Y. (2020). "Fabrication of all-cellulose nanocomposites from corn stalk," Journal of the Science of Food and Agriculture 100(12), 43904399. DOI: 10.1002/jsfa.10476

Dong, X. M., and Gray, D. G. (1997). "Induced circular dichroism of isotropic and magnetically-oriented chiral nematic suspensions of cellulose crystallites," Langmuir 
13(11), 3029-3034. DOI: 10.1021/la9610462

Escursell, S., Llorach, P., and Roncero, M. B. (2021). "Sustainability in e-commerce packaging: A review," Journal of Cleaner Production 280. DOI:

10.1016/j.jclepro.2020.124314

Gindl, W., and Keckes, J. (2005). “All-cellulose nanocomposite,” Polymer 46, 1022110225. DOI: 10.1016/j.polymer.2005.08.040

Hai, L. V., Kim, H. C., Kafy, A., Zhai, L., Kim, J. W., and Kim, J. (2017). “Green allcellulose nanocomposites made with cellulose nanofibers reinforced in dissolved cellulose matrix without heat treatment," Cellulose 24(8), 3301-3313. DOI: 10.1007/s10570-017-1333-7

Higgins, H. G., Stewart, C. M., and Harrington, K. J. (1961). "Infrared spectra of cellulose and related polysaccharides," Journal of Polymer Science 51(155), 59-84. DOI: $10.1002 /$ pol.1961.1205115505

Huang, J. W., and Li, Z. (2012). X-ray Diffraction of Polycrystalline MaterialsExperimental Methods, Principles and Applications, Metallurgical Industry Press, Beijing.

Jayme, G., and Knolle, H. (1964). "Introduction into empirical X-ray determination of crystallinity of cellulose materials," Papier 18, 249-255.

Kargarzadeh, H., Ahmad, I., Thomas S., and Dufresne A. (2017). Handbook of Nanocellulose and Cellulose Nanocomposites, Wiley 1, 58, Weinheim, Germany.

Li, Q., Chen, W., Li, Y., Guo, X., Song, S., Wang, Q., Liu, Y., Li, J., and Yu, H. (2016). "Comparative study of the structure, mechanical and thermomechanical properties of cellulose nanopapers with different thickness," Cellulose 23(2), 13751382. DOI: $10.1007 / \mathrm{s} 10570-016-0857-6$

Liang, C. Y., and Marchessault, R. H. (1959a). "Infrared spectra of crystalline polysaccharides. I. Hydrogen bonds in native celluloses," Journal of Polymer Science 37(132), 385-395. DOI: 10.1002/pol.1959.1203713209

Liang, C. Y., and Marchessault, R. H. (1959b). "Infrared spectra of crystalline polysaccharides. II. Native celluloses in the region from 640 to $1700 \mathrm{~cm}^{-1}$," Journal of Polymer Science 39(135), 269-278. DOI: 10.1002/pol.1959.1203913521

Manjunath, B. R., and Peacock, N. (1972). "Chain conformations in cellulose I and cellulose II," Applied Polymer 16(5), 1305-1308. DOI: 10.1002/app.1972.070160520

Mtibe, A., Linda, Z., Linganisoa, L. Z., Mathew, A. P., Oksman, K., John, M. J., and Anandjiwala, R. D. (2015). "A comparative study on properties of micro and nanopapers produced from cellulose and cellulose nanofibers," Carbohydrate Polymers 118, 1-8. DOI: 10.1016/j.carbpol.2014.10.007

Nelson, M. L., and O'Connor, R. T. (1964a). "Relation of certain infrared bands to cellulose crystallinity and crystal latticed type. Part I. Spectra of lattice types I, II, III and of amorphous cellulose," Journal of Applied Science 8(3), 1311-1324. DOI: 10.1002/app.1964.070080322

Nelson, M. L., and O'Connor, R. T. (1964b). "Relation of certain infrared bands to cellulose crystallinity and crystal lattice type. Part II. A new infrared ratio for estimation of crystallinity in celluloses I and II," Journal of Applied Polymer Science 8(3), 1325-1341. DOI: 10.1002/app.1964.070080323

Nishiyama, Y., Langan, P., and Chanzy, H. (2002). "Crystal structure and hydrogen bonding system in cellulose I $\beta$ from synchrotron X-ray and neutron fiber diffraction," Journal of the American Chemical Society 124, 9074-9082. DOI: 10.1021/ja037055w Oh, S. Y., Yoo, D. I., Shin, Y., Kim, H. C., Kim, H. Y., Chung, Y. S., Park, W. H., and 
Youk, J. H. (2005). "Crystalline structure analysis of cellulose treated with sodium hydroxide and carbon dioxide by means of X-ray diffraction and FTIR spectroscopy," Carbohydrate Research 340(15), 2376-2391. DOI: 10.1016/j.carres.2005.08.007

Park, S., Baker, J. O., Himmel, M. E., Parilla, P. P., and Johnson, D. K. (2010). "Cellulose crystallinity index: Measurement techniques and their impact on interpreting cellulose performance," Biotechnology for Biofuels 3, 1-10. DOI: 10.1186/1754-6834-3-10

Segal, L., Creely J. J., Martin Jr. A. E., and Conrad C. M. (1959). “An empirical method for estimating the degree of crystallinity of native cellulose using the X-ray diffractometer," Textile Research Journal 29(10), 786 -794. DOI: 10.1177/004051755902901003

Wang, H. Y., Li, S. Y., Wu, T. T., Wang, X. X., Cheng, X. D., and Li, D. D. (2019). “A comparative study on the characterization of nanofibers with cellulose I, I/II, and II polymorphs from wood," Polymers 11(1), 153. DOI: 10.3390/polym11010153

Wang Y. P., Wang G., Cheng H. T., Tian G. L., Liu Z., Xiao Q. F., Zhou X. Q., Han X. J., and Gao X. S. (2010). "Structures of bamboo fiber for textiles," Textile Research Journal 80 (4), 334-343. DOI: 10.1177/0040517509337633

Wang, X. J., Tong, W., Li, W., Huang, H. M., Yang, J., and Li, G. X. (2006). "Preparation and properties of nanocomposite of poly(phenylene sulfide)/calcium carbonate," Polymer Bulletin 57(6), 953-962. DOI: 10.1007/s00289-006-0652-x

Yue, Y. Y. (2011). A Comparative Study of Cellulose I and II and Fibers and Nanocrystals, Master's Thesis, Louisiana State University, Baton Rouge, USA.

Yukako, H., Eiji, T., and Tetsuo, K. (2017). "Characterization of individual hydrogen bonds in crystalline regenerated cellulose using resolved polarized FTIR spectra," ACS Omega 2(4), 1469-1476. DOI: 10.1021/acsomega.6b00364

Zavadskii, A. E. (2004). "The x-ray diffraction method of determining the polymorphous composition of cellulose for textured materials," Fibre Chemistry 36(2), 425-430. DOI: 10.1007/s10692-007-0106-8

Zhao, J. Q., He, X., Wang, Y., Zhang, W., Zhang, X., Deng, Y., and Lu, C. (2014). "Reinforcement of all-cellulose nanocomposite films using native cellulose nanofibrils," Carbohydrate Polymers 104(15), 143-150. DOI: 10.1016/j.carbpol.2014.01.007

Article submitted: June 17, 2021; Peer review completed: August 2, 2021; Revised version received: September 23, 2021; Further revised version received and accepted: October 27, 2021; Published: October 29, 2021.

DOI: $10.15376 /$ biores.16.4.8353-8365 